Instituto de Anatomia-Palólógica

del Hospital de Niños "M. Arriarán".

\title{
ULCERA CRONTCA DEL DUODENO EN EL LACTANTE
}

\author{
Por el Dr. JULIO ESPINOZA s.
}

Durante mucho tiempo se ha estimado por diversos autores que la úlcera crónica del ducdeno no existe en el lactante y que es muy rara en el niño mayor. Así lo afírman, por ejemplo, Finkelstein, Kundrat y Theile. Naturalmente que esta opinión está apoyada en la escasa o nula sintomatología a que da lugar esta lesión en el niño o a la completa atipia de los sintomas que corresponden a este cuadro on los primoros lustros de la vida. La observación clínica de úlceras crónicas gastro-duodenales en la pubertad y adolescencia. con una antigua historia de padecimientos digestivus que st remontaba a la primera infancia, así como al hallazgo casual de úlceras crónicas en cadáveres do niños menores. han sido causas suficientes para rectificar hoy día, hasta cierto punto, aquella antigua creencia y aceptar la efectiva existencia de este cuadro patológico en individuos de toda edad, incluso en el lactante.

En la literatura extranjera hemos encontrado que Guthrie, en 1942 publicó varios casos de úlcera gastro. duodenales agudas y crónicas en nin̄os, una de estas últimas en uno de 2 años; Gutman presentó a la Sociedad de Pediatria de París un caso de úlcora gástrica en un lactante de 8 moses; Berglund, en material de autopsias, encuentra úlceras en el $1.54 \%$ en niños por debajo de 1 año, y Schmidi. en idéntico material, las ha observado en el $1.8 \%$. Es necesario hacer la salvedad que los dos últimos autores se refiercn conjuntrmente a úlceras agudas y crónicas. En nuestro país. los colegas González Rodríguez y Prat Echaurren. 
en 1940, y Alliende y Raffo, en 1944, presentaron a esta Sociedad 4 casos de úlceras gastro-duodenales en niños de 11 a 15 años de edad.

En nuestro material de autopsias, cuyo número alcanza a 2,500 más o menos, de los cuales 1,500 corresponden a niños hasta de 1 año de edad, bemos podido observar sólo 2 casos de úlcera crónica del duodeno, Ja primera en

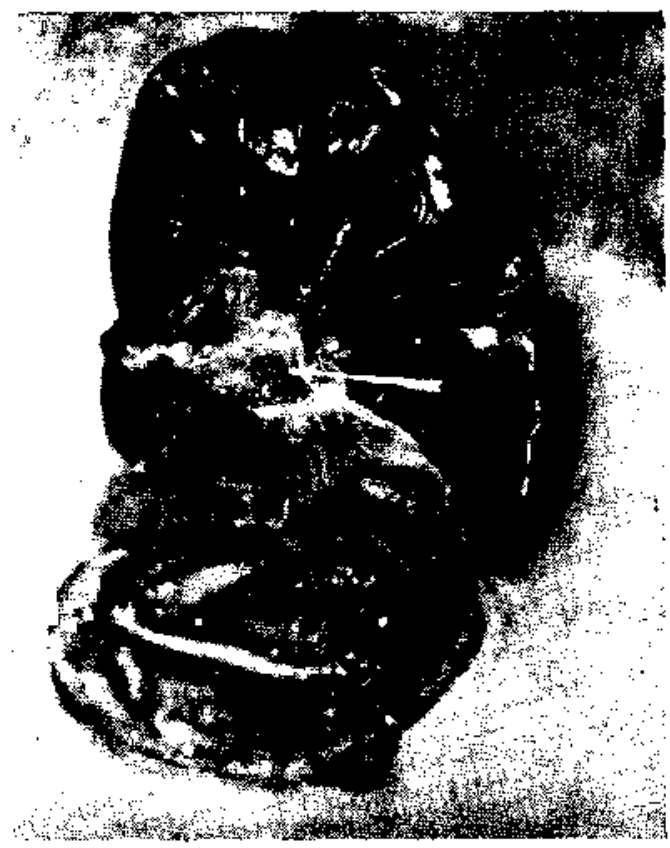

MACROFOTOGR AFIA

La flecha señala el sitio preciso de ia úlczra de 4 mms. de diámetro en la cară posterior del duodeno.

un lactante de 5 meses y la segunda en uno de 3 meses. ambos de sexo femenino.

El único móvil de esta presentación es dar a conocer estos dos casos nuestros como una contribución a la casuística nacional y una prueba más de la efectiva realidad de la lesión que comentamos. Agregaremos también algunas consideraciones clínicas a propósito del segundo caso. 
PRYMER CASO (I): Rosa S. - Obs. Cl, $\mathrm{N}^{*} 409$ - De 5 mesis de edad.

Ingresa al Hospital "M. Atriatán' el 3.0 de enero de 1944. Sin antectdenţas hereditarios ni personales de importancia. Permanece 14 dias afecta de enterooolitis y fallece al término de ellos de la misma afección.

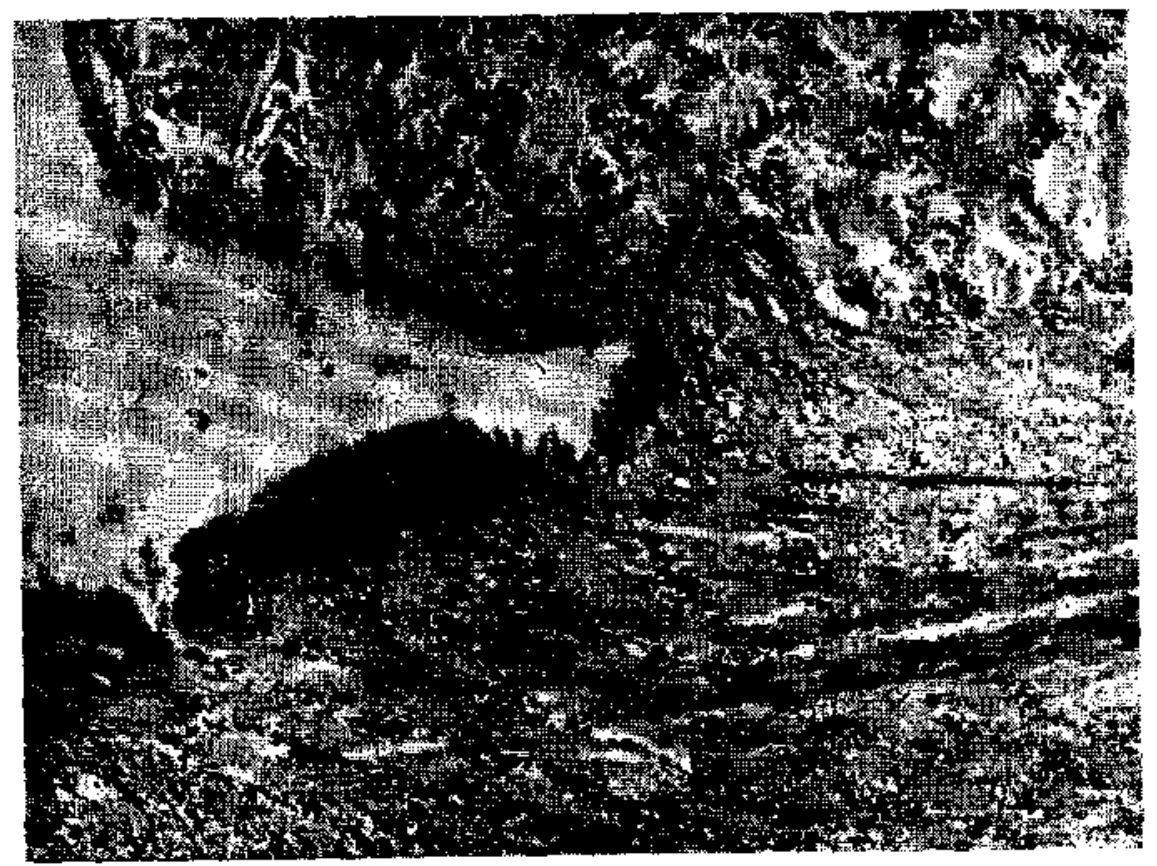

\section{MICRICFOTOGRAFIA No 1}

X. 100.-Hematoxilina-Eosina, $\rightarrow$ A la derceba se abservan las capas musculares que se resuelpen en pequeños haces al llegar al fondo de la ńlcera y ser aftectados por ella. Infiltrado inflamatorio difuso de casi todas las capas del órgano.

Necrosis superficial del tejido granulatorio.

En da autapsia $\left(N^{\circ} 82 / 44\right)$ se comprueba ила intensa enterocolitis ae:crótico-fibrinosa superficial, degeneración gránulo-grasosa marcada de los paréraquimas y una úlcera dnodenal que pasamos a describir macro y micros. cópicamente.

Duodeso: En su cara posteriot y a 8 mms. mas o menos del esfinctr pilórico, se observa una pequeña úkera ovoídea, que mide $4 \mathrm{mms}$. en su diámetro mayor, de fondo liso y de color blanquecino (var macrofotografia).

(1) Este caso faé presentado a la Socicdad de Atatomía Normal $r$ Patológica, en sesión del 30 de junio de 1948. 
Examen bistológico de la úlǫra duodetal (Micrefotografias Nos. $1 y$ 2): Ulcera que compromete basta las capas musculares inclusive, cuyo fondo se obsitva constituído por tejido granulatorio crónico con pequeños yasos de neoformacion $y$ elementos celulares linfocito-histiocitarios que infiltran tam. bién las regiones vecinas. Superficialmence el tejido granulatorio presenta una delgada capja dz necrosis. No se observa fibtosis (úlceta crónica).

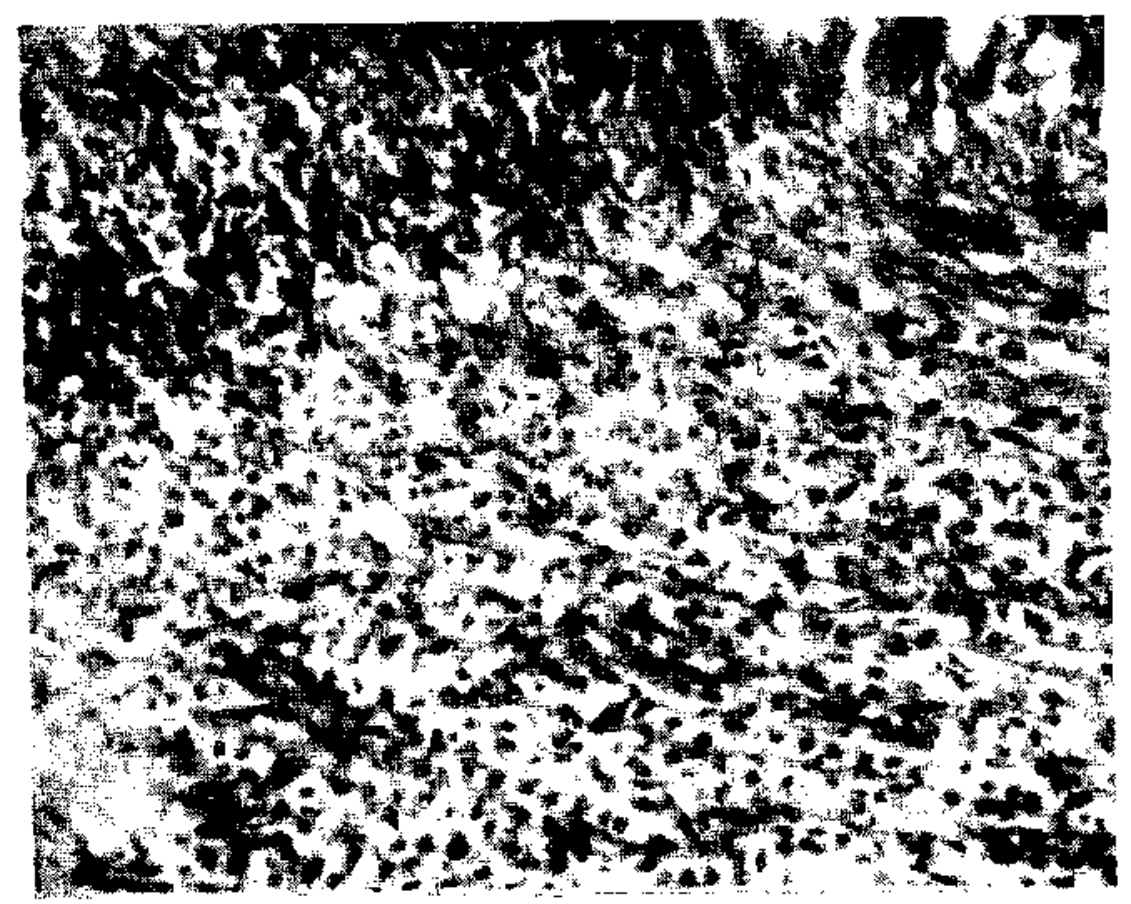

\section{MICROFOTOGRAFIA $\mathrm{N}^{\circ} 2$}

X. 180.--Hematoxilina-Eosia.--Tejido granulatorio crónico inespecífico rico en elementos linfocito-histiocitarios. Ausencia completa de polinucleares.

\section{SEGUNDO CASO: Blanca A. G. - Obs. 42203.}

Ingresa al Hospitad "M. Arriarán" el 21 de mayo del presente año a los 2 meses de edad. Sin antecedentes beriditarios de importancia. Nació de término y parto normal. Desde el nacimierto tiene vómitos explosivos abundantes, luago de comar at pecho. Deposiciones diatias. pero mup escasas. Ha consultado médico particular en varias oportunidades, quien ha prescrito sedances, pero sin ningún resultado. 
En tel examen físico se aprecis an lactante con mal estado genjeral. Pesa $3.300 \mathrm{kgrs}$, Abdomen de paredes delgadas, depresibles a indoioras, sín qui se palpe nada especial.

Al día sigujente de su ingreso se le practica radioscopia de estómago. informándose lo siguiente: "Estómago de forma, posición y tamaño normales.

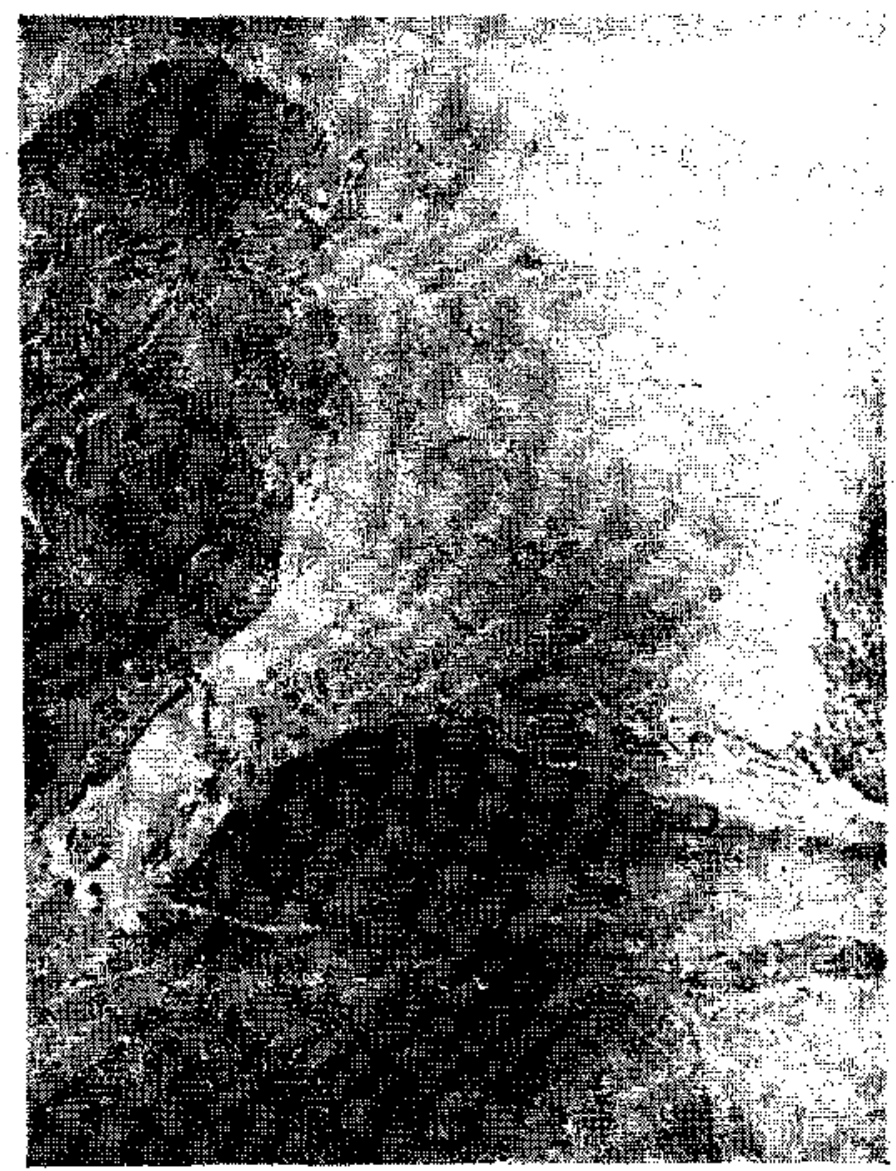

\section{MICROFO'FOGRAFIA $N^{-3}$}

X. 30.--Hematoxilina-Eosina.-Ulcera yuxtapilórica, El píloro a la izquierds. Su fondo esta sobre el hígado.

Buen tonus. peristaitismo y pasaj: pilórico. Bulbo duodeval en posición pos7. tior, normal". Control radioscópico a las 5 horas de ingerido el bario: "Aproximadamente un tercio de bario permanece en el estómago, el resto en al intestino delgado, de dactetes normabes". 
L. curva de piso observada en 5 dias es francamente descendeate; baja entre 20 y 30 grs: diarios, pese al tratamiento antiespasmódico que se le administra desde et ingreso.

Se diagnostica pitoroespasmo $y$ se opera el 31 de mayo.

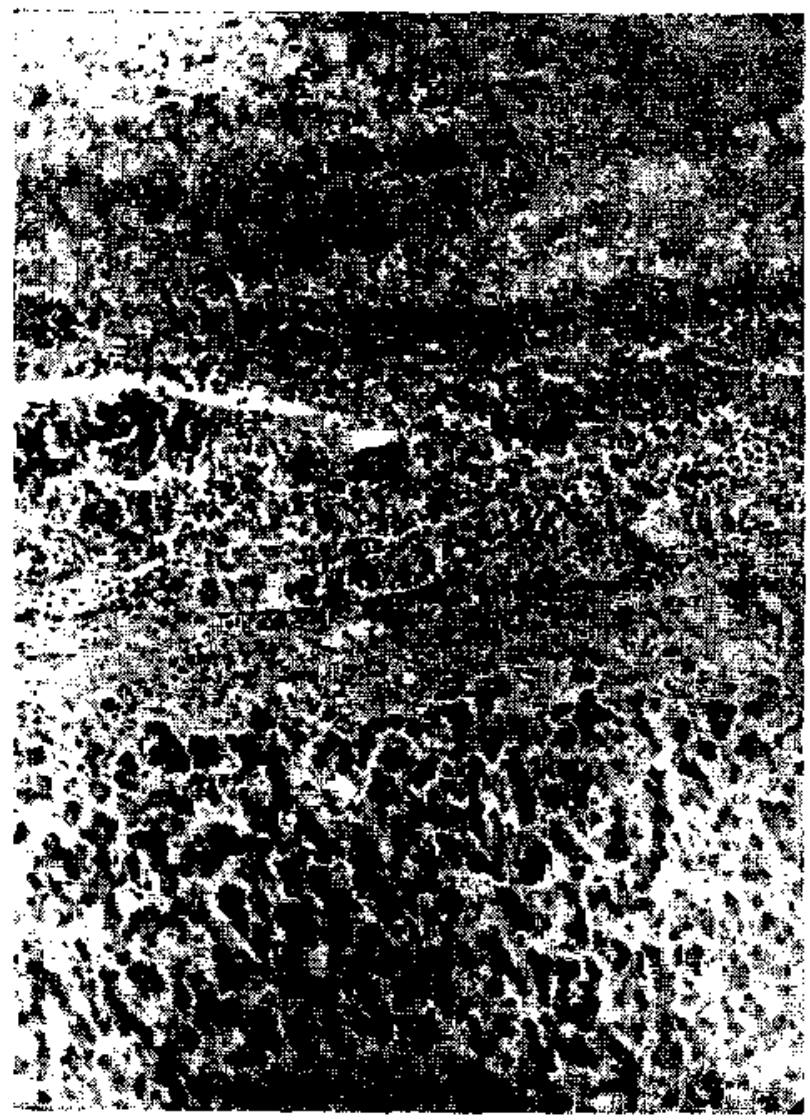

\section{MLCROFOTOGRAFIA $\mathrm{N}_{4}$}

X. 80.-Hematoxilina-Eosina.-Sobre el bigado se abserva tejido granulatotio rico en elementos redondos divzrsos y vasos sanguineos neoformados.

Se practica laparotomía supra umbilical parantetal derecha, se exterioriza el segmento pilórico $y$ en él no se comprueba tumoración alguna. Sobre ese segmento se hack una incisión con bisturi basta al plano mucoso. Se cierra por planos $y$ se pone montura $e$ hilo en la piel.

La niña sigue vomitando más o metos como lo hacía antes de la ape. ración. Se agrega, a partir de] $0^{*}$ dia de operada, una eventración de las 
asas intestinates y del estónago, por abertura de la berida operatoria, lo que se sepite dos veces mas, con cuatro dias de intervalo, La enfermita continúa decayendo $y$ vomitando, se torna grave y fallace al mes de operada.

En la autopsia se encontró nna niña muy enfilaquecida, caquéctica, con la berida operatoria abierta, supurada $y$ en el fondo de ella una asa intestinal fisculizada, que se vió despures que correspondía al yeyano a 1.10 m. del angulo duodenoryeyunal. Se encontró, además, una peritonitis crónica fibrinosa f fibro-adbesiva generalizada.

El estómago se encontraba con escatso tíquido gris-obscuro $y$ con sus plizguids bien marcados. El piloco no presentaba alteraciogies macroscópicas. En el duodeno se observaba na úlcera, ubicada en la ara posterior y casi junto al esfinter. Su forma era ovoidea y su diametro mayor media 5 mms. El fondo de la úleta eta. liso y de color blanquecino. El duodeno adhería firmemente por su cara posterior a la cata inferior del bigado (inicrofotografiz $N^{9} 3$ ).

El examen bistológico de la úlceta dió el siguiente resultado (microfotog tafia $\mathrm{N}^{2}$ 4). La úlcera compromede todas las capas del ótgano, tenitondo su fondo sobre la superficie bepática. En el fondo se aprecia la cápsula de G'isson engrosada e infiltradz por regalar cantidad de elementos inflamatorias mononualkados: linfocitos histiocitos y células plasmáticas. Sobre esta lámina conjuntiva $\mathbf{z}$ observa ona gruesa capa de a'specto fibrinoso, homogé. nea $y$ sin infilltrados celulares. En la parte más profunda de esta capa se observan capilates de neoformación, que proxdentes de la cápsola conjuntival del hígado, penetran a ésta (organización incipinto de la materia coagulada). En los bordes de la úicem se obiserva en forma manifiesta an tejido grantalatorio crónico inespecifico. En el corte se aprecia nítidamente que la whoera es yuxtapilórido (úlç̣ra crórica duadenal).

\section{Comentarios.}

El hallazgo de dos úlceras crónicas duodenales en 2,500 autopsias de niños practicadas, nos revela la rareza de este tipo de lesión, pero constituyen, al mismo tiempo. una prueba irrefutable de su existencia en los primeros meses de la vida. En el segundo caso llama, además, la atención la sintomatología que presentaba el lactante desde su nacimiento, lo que hace suponer, con grandes probabilidades de verosimilitud, que todo el cuadro sintomático faé debido a la úlcera del duodeno. Apoya esta manera de pensar la negetividad de varios signos físicos para aceptar el diagnóstico de píloroespasmo y la ninguna influencia que la operación practicada tuvo sobre el síntoma vómito. Palmer, citado por Finkelstein, asegura en un artículo publicado en 1921, que el vómito pertinaz, muy parecido al 
del píloroespasmo, es un sintoma observado ya muy frecuentemente en la úlcera duodenal.

Estos casos que comentamos tienen, además, un interés muy especial en lo que se relaciona con la patología de la úlcera, cuyos diferentes mecanismos habría que aplicarlos a un individuo recién nacido o aun en la vida fetal. pues, a juzgar por los resultados histopatológicos, probablemente en esa época ha empezado a producirse la lesión ulcerose. En este sentido tal vez tendríamos que aceptar que nuestros conocimientos sobre estas matorias están aún lejos de alcanzar a darnos una compresión clara y total del problema.

\section{Resumen.}

Se relatan dos casos de úlcora crónica del duodeno, comprobadas histológicamente en lactantes de 5 y 3 meses, tespectivamente $\mathrm{y}$ se hacen algunas consideraciones respecto de su frecuencia en la literatura nacional y extranjera.

\section{Summary.}

Two cases of chtonic ulcers of duodenum histologically confirmed are studied in infants of five and three months of age, respectively and some considerations are made with reference to their frecuency in national and foreing literature.

\section{Bibliografia.}

ALIIENDE J, y RAFFO J, - Caso elínico. Rev. Ch. de Pediatria. Año $\mathrm{XV} . \mathrm{N}^{0}$ 5, mayo 1940, pág. 400.

BRENNEMANN. - "Practice of Pediatrics". Vol. lil. 5: 21.

DONOVAN E. and SANTULLI. T. _- "Gastro-Duodenal Ulcer in infants and childhood". Am. J. of Dis, Chiddren, 1945. 69: 1>6.

ESFINOZA J. y ALTAMIRANO J. - "Ulcera ctónica del duodeno en un ractante". Arch. Chilenos de Morfologia (en prensa).

FINKELSTEIN H. - "Tratado de las enfermedades del niño de pecho". Ed. Labor. 1929.

GONZALEZ R. y PRAT E. - "Ulceras gastroدduodenale's en nüouos". Rev.

Cb. da Pediatria, junio 1940, fág. 363.

GUTHRIE K. J. - "Peptic alcer in infants and childhood. with a revition of the literature". Arch. of Dis. in cbildhood. June 1942.

WALMBERG E. - "Gastroduodenal ulaer in chifdhood". Acra Pedíatrica.

Vol. XXXII. Fas. 1, 31. 1945. page 86. 